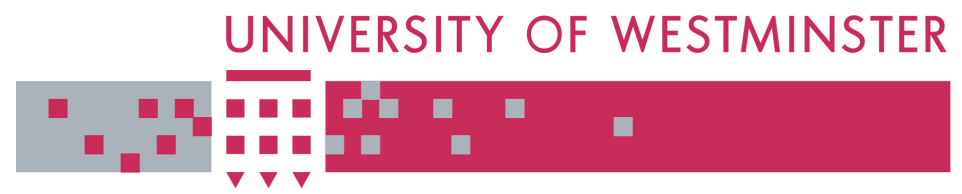

WestminsterResearch

http://www.wmin.ac.uk/westminsterresearch

\title{
Advanced satellite technologies for air traffic management
}

Panos Fines ${ }^{1}$

Ekaterina Christofylaki ${ }^{2}$

${ }^{1}$ Wireless Intelligent Systems Ltd., London

${ }^{2}$ School of Informatics, University of Westminster

Copyright (C) [2008] IEEE. Reprinted from 4th Advanced Satellite Mobile Systems: Proceedings, 26 - 28 Aug. 2008, Bologna, Italy. IEEE, pp. 55-59. ISBN 9781424421602.

This material is posted here with permission of the IEEE. Such permission of the IEEE does not in any way imply IEEE endorsement of any of the University of Westminster's products or services. Personal use of this material is permitted. However, permission to reprint/republish this material for advertising or promotional purposes or for creating new collective works for resale or redistribution to servers or lists, or to reuse any copyrighted component of this work in other works must be obtained from the IEEE. By choosing to view this document, you agree to all provisions of the copyright laws protecting it.

The WestminsterResearch online digital archive at the University of Westminster aims to make the research output of the University available to a wider audience. Copyright and Moral Rights remain with the authors and/or copyright owners.

Users are permitted to download and/or print one copy for non-commercial private study or research. Further distribution and any use of material from within this archive for profit-making enterprises or for commercial gain is strictly forbidden.

Whilst further distribution of specific materials from within this archive is forbidden, you may freely distribute the URL of the University of Westminster Eprints (http://www.wmin.ac.uk/westminsterresearch).

In case of abuse or copyright appearing without permission e-mail wattsn@wmin.ac.uk. 


\section{Advanced Satellite Technologies for Air Traffic Management}

\author{
P. Fines \\ Wireless Intelligent Systems Ltd., \\ London, U.K. \\ panos_fines@btinternet.com
}

\author{
E. Christofylaki \\ School of Informatics, \\ University of Westminster, \\ London, U.K. \\ ekateric@wmin.ac.uk
}

\begin{abstract}
This paper presents the current limitations and possible future upgrades of satellite communication systems to satisfy the requirements of air traffic management using compact and low cost aeronautical terminals.
\end{abstract}

Keywords - satellite aeronautical communications, air traffic management, aeronautical propagation, diversity techniques, air interface design

\section{INTRODUCTION}

The forecasted increase in air traffic and demand for onboard services leads the research for advanced satellite based systems for air traffic management. Satellite-based systems show great promise but the choice of suitable functions for civil aircraft operation is still to be defined and the expected performance has to be assessed in an operational environment.

The work presented in this paper was conducted in a project co-funded by the European Commission within the Sixth Framework Programme: Airborne New and Advanced Satellite techniques and Technologies in A System Integrated Approach (ANASTASIA). The activities of ANASTASIA range from the elaboration of operational needs to simulations and flight trials with validated avionics architecture and key technologies. ANASTASIA will contribute to the increase in airspace capacity to support this considerable growth with a target safety level at least as good as or better than the current level. Different parts of the civil aviation industry are involved towards this objective.

\section{SATEllite Systems For Air TRAfFic Management}

For Air Traffic Management (ATM) communications via satellite to be a success, the communication link must be affordable and very reliable, offering voice and data communication services over the widest possible area. At the same time the aeronautical earth station (AES) equipment must be affordable and compact, utilizing the smallest possible antenna for minimum air resistance, so that both terminals and antennas could be installed even on the smallest aircrafts, helicopters, etc. The cheapest and smallest possible antennas are characterized by low gain (LGA). Currently the well established satellite aeronautical services, for example Inmarsat's Classic-Aero, Swift-64 and the SwiftBroadband (SBB) systems are at L-band utilizing the Inmarsat I-4 state of the art multi-beam geostationary (GEO) satellites [1]. Nearly all these systems offer services using AES with intermediate (IGA) and high gain antennas (HGA) which are larger, heavier and more expensive than the LGA. Hence, the main task of the study is to identify how systems like the SwiftBroadband (SBB) could offer the necessary quality of service for ATM via an AES and propose necessary extensions and solutions that could upgrade the service to ATM standards.

The application of LGA results in a low receiver figure of merit $\mathrm{G} / \mathrm{T}$ which in turn results in a limited $\mathrm{C} /$ No available margin even in unfaded operating scenarios. The LGA wide beam-width also results in a low Carrier to Multipath $(\mathrm{C} / \mathrm{M})$ factor which in turn demands an increase of the fading margin required [2]. At high elevation angles (e.g. above $20^{\circ}$ ) both the minimum $\mathrm{C} / \mathrm{No}$ and fading margin requirements can be satisfied with the current SBB air interface and a reliable link in terms of forward error correcting (FEC) packet error rate (PER) can be established. At lower elevations (e.g. down to $5^{\circ}$ ) the $\mathrm{C} / \mathrm{M}$ decreases and these requirements become more difficult to be satisfied limiting the operating range, capacity and coverage area of the system. For a GEO satellite, AES operation from $20^{\circ}$ down to $5^{\circ}$ elevation results in about $30 \%$ service area extension. Therefore, in order to maximize the satellite service area, the AES must provide a reliable link even at low elevations. There are many ways around this problem:

Increase the number of satellites: As the satellite footprints increasingly overlap, the AES can switch between satellites at higher elevations. This is a very expensive solution and does not solve the reduced coverage at the earth's poles.

More EIRP power from the satellite: This is again a very expensive solution and already the available satellites have allocated the maximum power for aeronautical services. Moreover, more satellite power does not help to reduce the fading margin required since the $\mathrm{C} / \mathrm{M}$ factor remains unchanged.

More power efficient waveforms in the air interface: State of art modulation and coding techniques used in deep space applications have been employed and tested [3]. However, this is an expensive solution since any increase in power efficiency results in a reduction in the bandwidth efficiency and the overall system capacity. Furthermore, a system upgrade is 
needed involving both ground stations and aeronautical terminals.

More robust waveforms: The main difference between this option and the previous one is that the new waveforms improve the system capacity since they do not exchange the bandwidth efficiency for power efficiency as the power improvement is obtained from diversity gain [4]. Of course, system upgrade is needed involving software upgrades in both ground stations and aeronautical terminals.

Improve the aeronautical antenna: This option concerns only the AES and therefore, it is not so expensive since the satellite system and ground stations remain unchanged. For example, one could employ more than one LGA.

In this study we consider the last two options mentioned above. The current satellite systems do not allow for any significant diversity gain because priority was given in low system latency and simplicity of the terminals. However, without any changes it appears that the serviceable area is limited by the use of the LGA as explained previously. In this study we explore possible AES configurations to benefit from two types of diversity not previously utilized:

Time diversity: Extending the air interface to include new waveforms with FEC blocks beyond the current definitions, e.g. from $20 \mathrm{~ms}$ to $80 \mathrm{~ms}$ long. This requires a change in the air interface and software upgrades on both the ground station and the aircraft terminal.

Space diversity: Using the current air interface and employing a second LGA antenna at the AES. Only the aircraft terminal is affected.

The above two techniques provide quite independent diversity gains and one could select to employ one of the two or even both to maximize the diversity gain of the satellite system and the serviceable earth area.

\section{ANTENNA DIVERSITY}

For obtaining receivers with antenna diversity gain, their antenna pattern should aim at providing signals with similar long and short term statistics but also waveforms with very low correlation before they are combined for detection.

The antenna gain pattern is in general a complex vector and three methods are available for controlling its amplitude, phase and polarization as shown in Fig. 1. Each method is a specialized form of antenna pattern diversity [4]:

Space diversity: Identical antenna patterns are displaced in space by some distance.

Angle diversity: It involves identical antenna patterns in both polarization and phase but with different power profile orientation. Ideally the patterns can be displaced in the pointing angle.

Polarization diversity: It involves identical antenna patterns but with different polarizations.

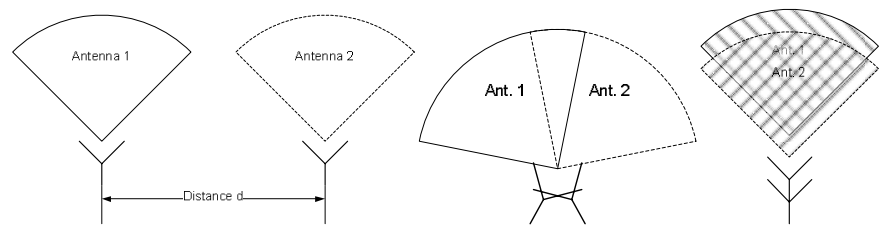

Figure 1. Antenna diversity: Space, Angular and Polarization

In the ANASTASIA context we consider AES with LGA with nearly omni-directional pattern. Therefore, space diversity can be more easily achieved than polarization or angle diversity. When the incident wave distribution covers large angles then it also has a large angular spread causing large Doppler spread. In this case a small change of phase between patterns has a strong effect on decorrelating the received multipath signals. The next issue is to identify the appropriate combining method [4].

\section{COMPARISON OF ANTENNA COMBINING METHODS}

Simple antenna switching (Fig. 2) may not perform as well as selection combining (Fig. 3) since the diversity gain depends on the choice of the SNR switching threshold. The selection combining method measures the SNR from the two antennas and always selects the stronger.

For a two antenna diversity scheme, the diversity gain difference between simple adding (Fig. 4) and maximum ratio combining (Fig. 5) methods is small and in practice it may become even smaller if the weighting factors in the combining method are not continuously correctly estimated. However, for more than two antennas the diversity gain difference is much greater. In the ANASTASIA context we are considering two antennas only and the diversity gain difference turns out rather small (e.g. about $1 \mathrm{~dB}$ ). Therefore, equal gain combining offers most of the performance gain without having to accurately estimate the weights and multiply the amplitudes before addition.

Finally, a two terminal method is presented (Fig. 6), in which the higher protocol layers receive demodulated and decoded packets from two AES but always select at most one after checking the packet integrity, for example using checksum (CRC) verification. This method may seem expensive since it involves some terminal duplication, but in practice extensive development may not be needed.

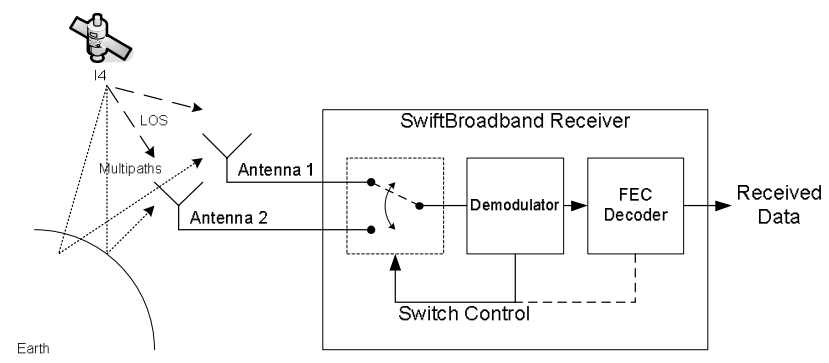

Figure 2. Antenna swiching 


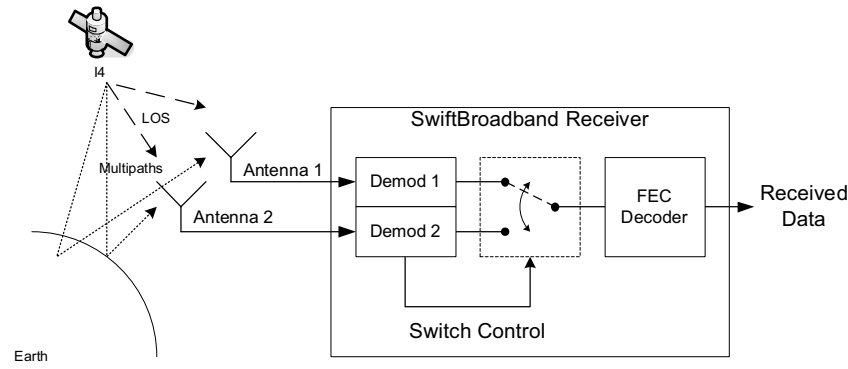

Figure 3. Antenna selecting method

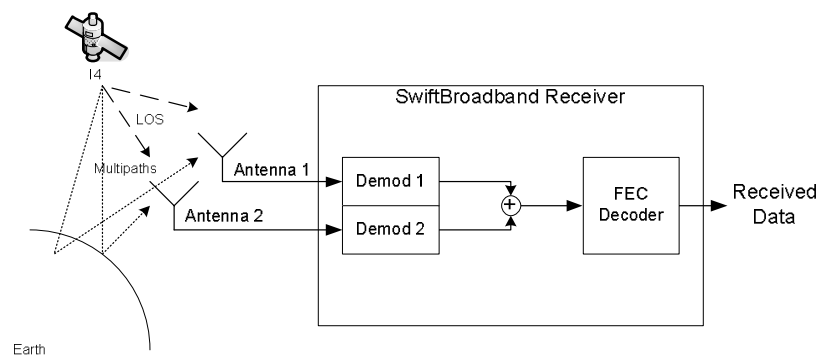

Figure 4. Fixed combining method

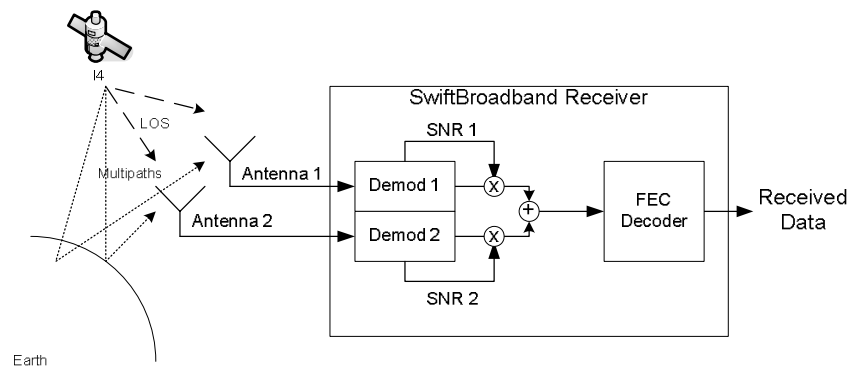

Figure 5. Maximum ratio combining method

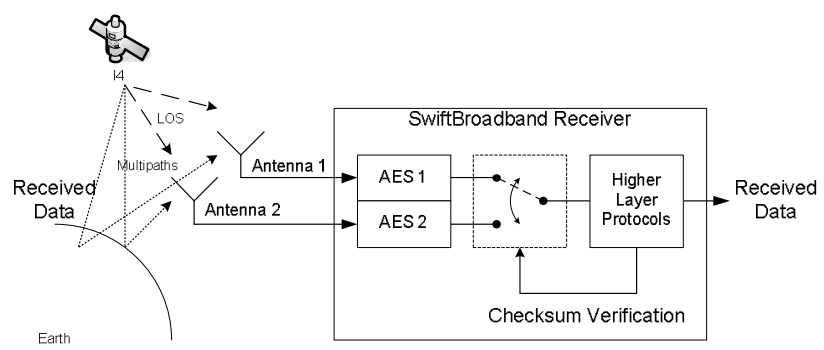

Figure 6. Receiver combining method

How the signals of the two antennas are combined using the different methods is shown in Fig. 7. The maximum selection algorithm switches between the antennas and provides as output a signal no better than the stronger of the two inputs. When the two branches are equal both the maximum ratio and the fixed gain combining algorithms provide a diversity gain up to $3 \mathrm{~dB}$. When the two branches differ considerably in gain (e.g. one of the two antennas goes through a deep fade), the fixed gain algorithm provides as output the best of the two reduced by up to $3 \mathrm{~dB}$, where the maximum ratio combiner provides an output no worse than the best branch.

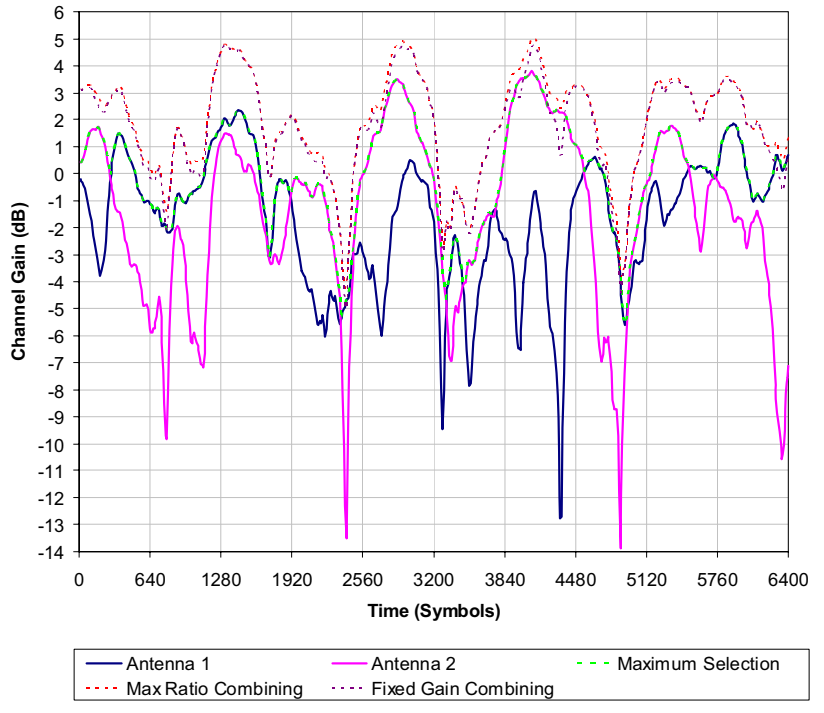

Figure 7. Resulting signal from different comining methods

Clearly the maximum ratio combiner is a winner but at the same time it is the most complex method since it requires accurate and continuous SNR estimation on each branch. If the SNR estimators are inaccurate, the fixed gain algorithm may be a very sensible fall back choice.

\section{Simulation Results and Performance}

A simulation model was implemented including SBB transmitters, the aeronautical propagation channel and an AES with one and two antennas combined with all the algorithms mentioned previously. Furthermore, two types of forward link transmitters were implemented: one with the currently used SBB waveform (F80T1QL8-4B) with 20 ms long FEC blocks and a second one with the new waveform (F80T1QL8-1B) with $80 \mathrm{~ms}$ long FEC blocks.

In the aeronautical satellite communication channel the received signal consists of a LOS component and several multipath components [2]. All the components are time varying and combined at the antenna with random phases and amplitudes, and therefore, they occasionally add up or cancel each other out. The resulting signal is therefore, subject to time varying fading characterized by the carrier to multipath power ratio $(\mathrm{C} / \mathrm{M})$ factor, the fading Doppler spread $(\mathrm{Fd})$ and some delay spread which is insignificant because only the narrowband SBB channels are considered. In the simulations an aeronautical channel has been assumed with parameters: $\mathrm{C} / \mathrm{M}=9 \mathrm{~dB}$ and $\mathrm{Fd}=30 \mathrm{~Hz}$.

The results to be presented in the following sections are expressed in terms of PER versus the available C/No. The SBB is a data packet oriented system and for efficient SBB operation, the SBB air interface should provide a communication link with PER $<10^{-3}$. The results include the following AES terminal and antenna configurations:

Single antenna AES in AWGN: This is the normal AES configuration consisting of one antenna and one AES. There is no aeronautical fading in the channel (i.e. AWGN channel) and 
this curve is taken as reference for all other configurations for measuring the fading margin required.

Single antenna AES with fading: This is the normal AES configuration consisting of one antenna and one AES. There is aeronautical fading in the channel (i.e. $\mathrm{C} / \mathrm{M}=9 \mathrm{~dB}, \mathrm{Fd}=30 \mathrm{~Hz}$ ) and this curve is taken as reference for measuring the diversity gain that may be provided using all the other configurations.

Two AES terminals: This configuration employs two independent AES with two uncorrelated antennas. The SBB higher layer protocols, after CRC verification, accept only one of the two physical layer packets.

Select Max: This configuration consists of two uncorrelated antennas and one AES that monitors the signal strength from both antennas and always selects the branch with the best SNR.

Fixed ratio combining: This configuration consists of two uncorrelated antennas, one AES equipped with two demodulators and a single decoder. The demodulator outputs are simply added before the decoder.

Maximum ratio combining: This configuration consists of two uncorrelated antennas, one AES equipped with two demodulators and a single decoder. The demodulator outputs are weighted according to their SNR and then added before the decoder.

\section{A. Space Diversity Results with current $S B B$ waveform}

Without any change in the SBB air interface but with a second antenna at the AES, the results are expressed in terms of PER versus the available C/No and are presented in Fig. 8. In the unfaded (i.e. AWGN) case, the single antenna AES configuration requires $\mathrm{C} / \mathrm{No}=45 \mathrm{dBHz}$ for achieving $\mathrm{PER}<10^{-3}$. This $\mathrm{C} / \mathrm{No}$ value is taken as reference for measuring the fading margin required for all other configurations. With aeronautical fading the single $\mathrm{AES}$ configuration requires about $\mathrm{C} / \mathrm{No}=53$ $\mathrm{dBHz}$ for achieving $\mathrm{PER}<10^{-3}$, meaning that $8 \mathrm{~dB}$ fading margin is required. This $\mathrm{C} / \mathrm{No}$ value is taken as reference for measuring the diversity gain for all other configurations. In the AWGN unfaded scenario the two antennas with Maximum or Fixed Ratio Combining methods shall perform $3 \mathrm{~dB}$ better than a single antenna case. For achieving PER $<10^{-3}$, the best diversity gain is $6.7 \mathrm{~dB}$ obtained by the 'Maximum Ratio Combining' method while very close at $6.3 \mathrm{~dB}$ stands the 'Fixed Ratio Combining' method. Both methods provide impressive diversity gains which in turn may result in improved link quality, system efficiency, capacity and coverage area improvements. In fact, all the combining methods gave noticeable diversity gains. The 'Select Max' method provides $3 \mathrm{~dB}$ diversity gain and the 'Two AES' method $3.6 \mathrm{~dB}$.
F80T10 8-4B Packet Error Rate Performance versus C/No for two Antenna AES

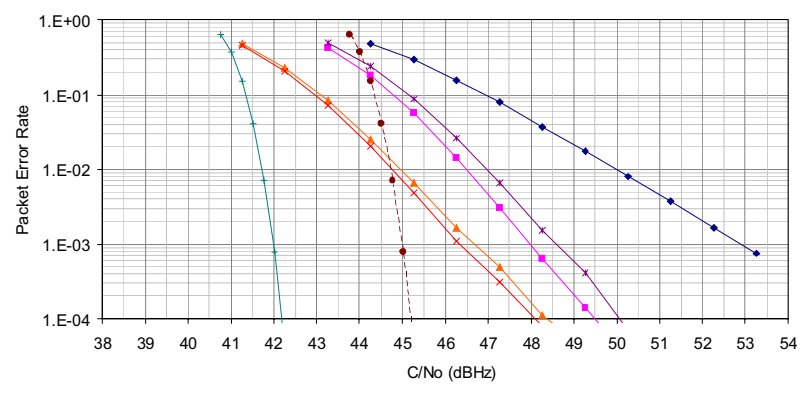

- F80T1QL8-4B C/M=9dB, Fd=30Hz Single Antenna - F80T1QL8-4B C/M=9dB, Fd=30Hz Select Max F80T 1QL8-4B C/M=9dB, Fd=30Hz Fixed Ratio Combining - - F8OTTQL8-4B CMM-9dB, Fd=3OHz Max Ratio Combinin F

F80T1QL8-4B AWGN Two Atnennas

Figure 8. Performance with antenna space diversity

\section{B. Time Diversity}

In this section it is assumed that the AES uses a single antenna and the SBB air interface which currently employs only $20 \mathrm{~ms}$ FEC blocks, is extended to include new waveforms with $80 \mathrm{~ms}$ long FEC blocks. The FEC coding type and rate has not been changed but new interleavers have been designed. Within these blocks some diversity gain is obtained by interleaving in time the channel symbols. The main implication on the SBB system's behavior is that the data latency has been increased from $20 \mathrm{~ms}$ to $80 \mathrm{~ms}$ (relative to the AES antenna). For real time voice and video streaming, the transmitter latency also increases by a similar amount. In all other aspects the new waveforms are totally compatible with the SBB air interface. Therefore, both current and new waveforms have the same signal in space and bandwidth characteristics, require the same satellite EIRP, but in addition the new waveform should have slightly better power efficiency in AWGN and it may require less fading margin due to the diversity provided by the longer FEC length.

Extensive simulations have confirmed a significant performance improvement as shown in Fig. 9. The simulation results have been obtained assuming an aeronautical propagation model with parameters: $\mathrm{C} / \mathrm{M}=9 \mathrm{~dB}$ and $\mathrm{Fd}=30$ $\mathrm{Hz}$.

In the AWGN unfaded scenario, the new waveform requires around $0.6 \mathrm{~dB}$ less $\mathrm{C} / \mathrm{No}$ than the current $\mathrm{SBB}$ waveform, since the turbo coding performance improves as the FEC block length increases. With aeronautical fading, the new waveform gain is even more significant: $4.3 \mathrm{~dB}$ reduction in the fading margin required for achieving PER $<10^{-3}$ compared to the current waveform. 
F80T1QL8-4B and F80T1QL8-1B Packet Emor Rate Performance versus C/No for single Antenna AES

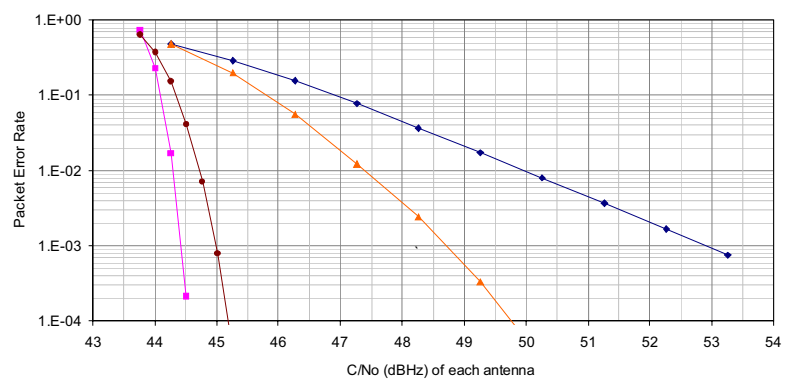

- F80T 1QL8-4B C/M=9dB, Fd=30Hz Single Antenna - - F80T1QL8-1B AWGN Single Atnenna

¿-F80T 1QL8-1B C/M=9dB, Fd=30Hz Single Antenna - F80T1QL8-4B AWGN Single Atnenna

Figure 9. Performance with time diversity

\section{Time and Space Diversity Results with the new waveform}

One can combine the benefits of time and space diversity. This can be achieved by using the new waveforms with $80 \mathrm{~ms}$ long FEC blocks, in a new terminal incorporating two antennas. The results are shown in Fig. 10. The best diversity gain is $9.1 \mathrm{~dB}$ obtained with the 'Maximum Ratio Combining' method but very close at $8.8 \mathrm{~dB}$ stands the 'Fixed Gain Combining' method. Both methods provide impressive diversity gains which in turn may result in significant system quality and capacity improvements. The 'Select Max' method provides $7.3 \mathrm{~dB}$ diversity gain and the 'Two AES' method 6.3 $\mathrm{dB}$. It should be noted that the assumption is that both antennas have visibility of the satellite and the incoming multipath components are mutually uncorrelated.

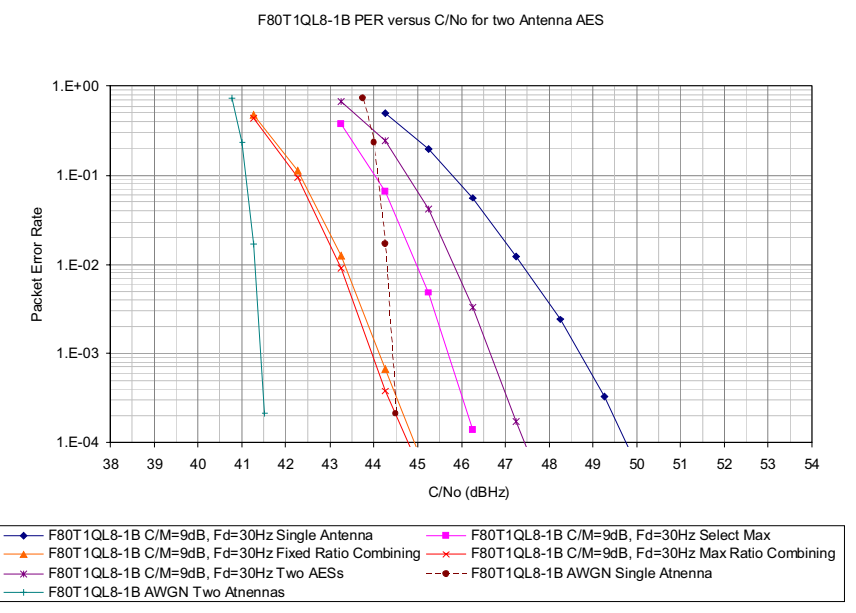

Figure 10. Performance with time and space diversity

\section{CONCLUSIONS}

The aim of this analysis is to extend the satellite coverage area of ATM. Extending the service area (e.g. by 30\%) implies that the AES should also operate at lower elevation angles (e.g. from $20^{\circ}$ down to $5^{\circ}$ ). This requires extensive fading margin not currently available for AES with a single LGA. We have presented the basic principles of time and space diversity and their potential benefits when applied to satellite systems accessed by an AES with LGA. Simulation results indicate the following gains:

Time diversity: Taking as reference the current SBB waveforms, a fading margin reduction of $4.3 \mathrm{~dB}$ has been obtained using time diversity achieved by extending the FEC blocks from 20 to $80 \mathrm{~ms}$ long.

Space diversity: A fading margin reduction of $6.7 \mathrm{~dB}$ has been obtained with space diversity using a second antenna and optimal combining at baseband.

Combining time and space diversity: $\mathrm{Up}$ to $9 \mathrm{~dB}$ reduction in the fading margin could be achievable. By using a second antenna other reception problems are potentially reduced: blockage of the satellite signal during banking or strong multipath reflection from the aircraft's structure.

The results indicate very significant gains but the working assumptions should also be noted: The two antennas are sufficiently separated in space so that the incoming multipaths are totally decorrelated and the diversity receiver has no implementation losses. In practice, some correlation between antennas and suboptimal receivers may reduce the diversity gain. The main outcome is that time and space diversity allows for more system capacity and very reliable links with AES with two LGAs down to $5^{\circ}$ elevation, covering around $38 \%$ of the earth with a single GEO satellite.

\section{REFERENCES}

[1] M. Richharia, E. Trachtman, P. Fines, "Broadband Global Area Network Air Interface Evolution", 23rd Communications Satellite Systems Conference, Organizer American Institute of Aeronautics and Astronauts (AIAA), Rome, September 2005.

[2] M. Richharia, N. Kaluvala, J. Axel, P. Fines, "Aeronautical Channel Model for Broadband L-Band Satellite Commuincations", 23rd Communications Satellite Systems Conference, Organizer American Institute of Aeronautics and Astronauts (AIAA), Rome, September 2005.

[3] P. Fines, E. Christofylaki, S. Papaharalabos, P. Febvre, "Low Rate Turbo Code Extensions and Modem Design for High Reliability Satellite Links", 4th ESA International Workshop on Tracking, Telemetry and Command Systems for Space Applications, ESOC Darmstadt, Germany, Sept. 2007.

[4] R. Vaughan and J. B. Andersen, "Channels, Propagation and Antennas for Mobile Commuincations", IEE publication 2003, ISBN 085296084 0 . 